\title{
Magnetic moment of iron in metallic environments
}

\author{
G. W. Fernando \\ Department of Physics, U-46, University of Connecticut, Storrs, Connecticut 06269; \\ Department of Physics, Brookhaven National Laboratory, Upton, New York 11973; \\ and Institute of Fundamental Studies, Hantana Road, Kandy, Sri Lanka \\ R. E. Watson and M. Weinert \\ Department of Physics, Brookhaven National Laboratory, Upton, New York 11973 \\ A. N. Kocharian \\ Department of Physics, California State University, Northridge, California 91330
}

\author{
A. Ratnaweera and K. Tennakone \\ Institute of Fundamental Studies, Hantana Road, Kandy, Sri Lanka
}

(Received 28 April 1999)

\begin{abstract}
Rare-earth iron nitrides are emerging as an important class of magnetic materials. In certain rare-earth iron compounds, the insertion of small atoms such as nitrogen and boron has resulted in significant changes in the magnetic properties in the form of higher Curie temperatures, enhanced magnetic moments, and stronger anisotropies. In an attempt to understand some of the above, we have focused on two nitride phases of $\mathrm{Fe}$, namely $\mathrm{Fe}_{4} \mathrm{~N}$ (cubic) and $\mathrm{Fe}_{16} \mathrm{~N}_{2}$ (tetragonal). For the $\mathrm{Fe}_{16} \mathrm{~N}_{2}$ phase, the average Fe moment reported by different experimental groups varies over a wide range of values, from $2.3 \mu_{B}$ to $3.5 \mu_{B}$. We will discuss some of the recent experiments and examine some related theoretical questions with regard to $\mathrm{Fe}$ having such an unusually large moment in a metallic environment. Employing a Hubbard-Stoner-like model in addition to local-density results, it is shown that an unusually large on-site Coulomb repulsion is necessary if one is to obtain a moment as large as $3.5 \mu_{B}$.
\end{abstract}

\section{INTRODUCTION}

Although a physical picture of quantum magnetism was developed many decades ago, there are still numerous open questions and unresolved problems with regard to understanding the microscopic mechanisms of ferromagnetism and strong ferromagnetic saturation. Two different theoretical approaches that have been introduced to examine these concepts are (i) band theory based on an effective single-particle picture, where the exchange-correlation splitting is introduced through a spin-dependent one-particle potential, as is commonly done within the local-spin-density approximation (LSDA) based on density-functional theory (DFT) and (ii) explicit inclusion of many-body effects through a "minimal lattice"' Hamiltonian with a few adjustable parameters such as the Hubbard model to understand the origin of ferromagnetism related to correlated electrons. Although the Hubbard model is usually associated with antiferromagnetism, it is possible to find conditions for ferromagnetic saturation in the metallic state. In contrast, DFT based approaches, at least in principle, are parameter free and the LSDA is well suited for describing itinerant magnetism.

The iron nitrides that are discussed in this paper were discovered ${ }^{1}$ more than forty years ago. In $1972, \mathrm{Fe}_{16} \mathrm{~N}_{2}$ thin films produced by evaporating iron in nitrogen were found to have unusually large saturation polarizations. ${ }^{2}$ Although these films did contain substantial amounts of $\alpha$-Fe, high polarizations were attributed to the presence of the nitride phase. Recent experiments ${ }^{3-10}$ related to measuring magnetic moments of iron in iron nitrides have raised various questions, both experimental and theoretical. Early evidence for unusually large moments associated with $\alpha^{\prime \prime}-\mathrm{Fe}_{16} \mathrm{~N}_{2}$ came from Mössbauer studies of thin films and small particles. ${ }^{11} \mathrm{~A}$ recent NMR study ${ }^{9}$ also tends to support the existence of a large site moment (of about $3.5 \mu_{B}$ ) and an average value of $\bar{\mu}_{\mathrm{Fe}}=2.9 \mu_{B}$ for the unit cell. However, there is a dissenting point of view, ${ }^{10,11}$ that suggests the possibility of an "egregious error' in the interpretation of data. Reference 10 points to the presence of a disordered $\mathrm{Fe}_{16} \mathrm{~N}_{2}$ phase $\left(\alpha^{\prime}-\mathrm{N}\right.$ martensite) in most of the samples studied.

In this paper we will examine some theoretical questions related to magnetism in metallic systems. This will be done through examining first principles as well as parametrized many-body approaches. One such question is whether band theory is capable of yielding such large moments for $\mathrm{Fe}$ in a metallic system. This should be addressed from several different aspects: first, whether the spin exchange and correlation effects included in a LSDA-type approach (and possible orbital contributions not included in LSDA) can yield such large magnetic moments and second, whether any mean-field theory can describe such situations or whether fluctuations are important. These are somewhat related questions, usually overlooked in most studies. We will argue that within a parametrized mean-field theory it is possible to obtain such large moments - though using somewhat unphysical parameters-while within the usual LSDA it is not possible. This is essentially indicating at least that the spin exchangecorrelation effects should be different, or treated more ex- 
plicitly as in Hartree-Fock (HF) or Hubbard approaches, in order to obtain large magnetic moments of the scale claimed experimentally. This statement makes at least partial sense since in the completely localized situation of an atom, HF predictions of the multiplet levels seem to correlate well with Hund's rule predictions and experiment. However, our focus is on metallic ferromagnets with long-range order, while a collection of noninteracting atoms would constitute a paramagnet, with individual atoms carrying large local moments; i.e., having local saturation at the atomic level alone is not sufficient to produce a strong ferromagnet. These issues are relevant to technologically important materials such as (bulk) permanent magnets, magnetic multilayers, clusters and low dimensional systems based on transition, and rare-earth and actinide elements.

There have been several first-principles LSDA band calculations for this system, using methods ranging from the atomic sphere approximation (ASA) based calculations ${ }^{14,15}$ to full potential ones. ${ }^{16}$ Our full potential linear augmented Slater-type orbital (LASTO) results based on LSDA are quite similar to those reported in Ref. 16. In an attempt to go beyond the LSDA, and to include more explicit correlations, a local-density-approximation (LDA) $+\mathrm{U}$ calculation has also been reported. ${ }^{17} \mathrm{LDA}+\mathrm{U}$ method is derived from the Hubbard model and has been used to describe transitionmetal oxides which are insulators. ${ }^{18}$ However, it is not clear whether such an approach using large $U$ values $(\simeq 4 \mathrm{eV})$ could be justified for these materials which are metallic. One open question is whether strong, saturated ferromagnetism in hard magnets based on a $3 d$ transition metal, can be understood reasonably well from the LSDA based band theory or from a Hubbard-type model with proper intraatomic interaction and hopping parameters. In this paper we report results from a variety of full potential LDA and LSDA calculations on these nitrides in addition to what might be termed Hubbard-Stoner calculations based on unpolarized LDA results.

\section{CRYSTAL STRUCTURES}

These iron nitrides are fascinating examples of magnetic solids, with a mixture of bcc- and fcc-like local Fe environments including some distortions. The ground-state crystal structure of $\mathrm{Fe}_{4} \mathrm{~N}$ is a fcc lattice of $\mathrm{Fe}$ atoms with a nitrogen atom occupying the body center position; i.e., each nitrogen atom is surrounded by an octahedron of Fe atoms. There are two inequivalent $\mathrm{Fe}$ sites here, one (Fe-II) being closer to nitrogen than the other $(\mathrm{Fe}-\mathrm{I})$. Fe-I and $\mathrm{N}$ sites have $O_{h}$ local symmetry while Fe-II has $D_{4 h}$. The (observed) lattice constant used for this fcc cell in our calculations is $3.80 \AA$. Figure 1 shows the crystal structure of $\mathrm{Fe}_{16} \mathrm{~N}_{2}$. In this structure, there are three inequivalent $\mathrm{Fe}$ sites, two of them closer to being in a bcc environment. We have labeled Fe-I as the site furthest from $\mathrm{N}$, while Fe-II being the closest to $\mathrm{N}$ (see Table I as well). $\mathrm{Fe}_{16} \mathrm{~N}_{2}$ can be thought of as alternate units of fcc $\mathrm{Fe}$ and $\mathrm{Fe}_{4} \mathrm{~N}$ units, with the atoms being allowed to relax (or having a unit cell consisting of eight distorted bcc units). The primitive cell here is a body-centered-tetragonal one, and the local symmetries of Fe-I, Fe-II, Fe-III, and N are $D_{2 d}, C_{4 \mathrm{v}}, C_{1 h}$, and $D_{4 h}$, respectively. The lattice con-

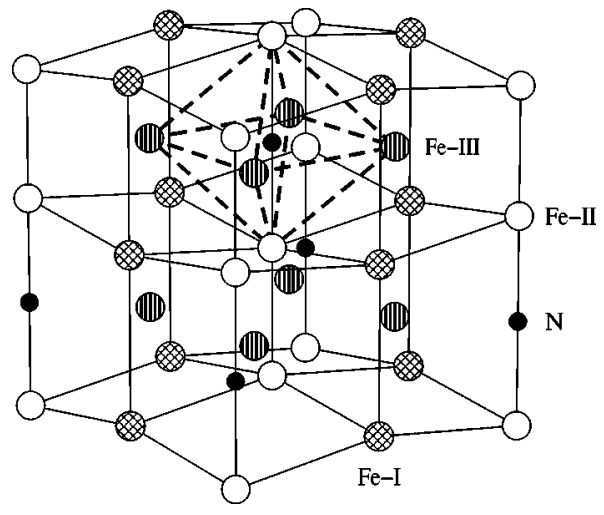

FIG. 1. Crystal structure of body-centered-tetragonal (bct) $\mathrm{Fe}_{16} \mathrm{~N}_{2}$. Note the octahedral environment around $\mathrm{N}$ and the three different $\mathrm{Fe}$ sites. The lattice constants used for the bct cell are $a$ $=5.72 \AA$ and $c=6.29 \AA$.

stants used for the body-centered-tetragonal $\mathrm{Fe}_{16} \mathrm{~N}_{2}$ cell are $a=5.72 \AA$ and $c=6.29 \AA$.

\section{EXPERIMENTAL BACKGROUND}

It is important to follow the experimental background in order to understand the theoretical motivation for this paper. There are several excellent recent reviews on the subject. ${ }^{7,11}$ In recent years a number of different experimental groups have been able to grow thin films that contain $\mathrm{Fe}_{16} \mathrm{~N}_{2}$, using techniques such as molecular beam epitaxy (MBE). The large polarization values $(2.9 \mathrm{~T})$ reported by the Hitachi group ${ }^{5}$ for thin films containing $\mathrm{Fe}_{16} \mathrm{~N}_{2}$ were the most notable due to their implications. It appears to be extremely difficult to obtain reasonably large samples of pure, singlecrystal $\mathrm{Fe}_{16} \mathrm{~N}_{2}$. This is a major reason for the controversial claims with regard to average magnetization and, hence, Fe moments. Magnetization measurements have been done on samples that contain several phases such as bcc Fe, fcc Fe, and $\mathrm{Fe}_{4} \mathrm{~N}$, in addition to $\mathrm{Fe}_{16} \mathrm{~N}_{2}$. Saturation magnetization $\sigma_{0}$ for such samples is always below the saturation value for pure, bcc iron. However, based on a phase analysis of the samples using either Mössbauer spectra or x-ray diffraction, it is possible to assign a $\sigma_{0}$ value for the $\mathrm{Fe}_{16} \mathrm{~N}_{2}$ phase. This value ranges from 225 to $310 \mathrm{emu} / \mathrm{g}$. ${ }^{7}$ The upper end of these values lead to predictions of higher average moments and hence to an unusually large value of moment $\left(\geqslant 3.5 \mu_{B}\right)$ for the $\mathrm{Fe}$ site that is furthest from nitrogen. A more recent X-ray-diffraction and Mössbauer study identifies the presence of disordered, octahedral $\mathrm{N}$ sites in a $\alpha^{\prime}-\mathrm{Fe}_{16} \mathrm{~N}_{2}$ phase that coexists with the ordered $\alpha^{\prime \prime}$ phase, and their saturation magnetization values for the ordered phase have an upperbound of about $240 \mathrm{emu} / \mathrm{g}$ at room temperature. ${ }^{10}$

The NMR work ${ }^{9}$ does not use this type of a phase analysis to deduce moments. It identifies the NMR frequencies (hence hyperfine fields) assigned to various sites and then relies on "proportionality constants" that relate hyperfine fields to the values of magnetic moments at those sites. Using reasonable values for these "constants," the NMR study appears to confirm the existence of a site moment that is $\simeq 3.5 \mu_{B}$. One important point of agreement among these experiments is the observation of a large hyperfine field (of about $41.8 \mathrm{~T}$ for the Fe-I site, compared to bcc $\alpha$-Fe value of $33.8 \mathrm{~T}$ ) for the 
TABLE I. Tables below list various sites of $\mathrm{Fe}_{16} \mathrm{~N}_{2}$ and $\mathrm{Fe}_{4} \mathrm{~N}$ and related information. The symbols bcc and fcc accompanying site labels denote bcc and fcc-like sites respectively. The symbol (?) for Fe-II in 16:2 indicates that its environment is neither bcc-nor fcc-like. Parenthesis in the last four lines indicate either the number of atoms at (or within) a given distance or the type of Fe atom. For comparison, some bcc Fe values are (i) LSDA and experimental moment $=2.2 \mu_{B}$ and (ii) Fe-Fe nearest-neighbor distance $=2.48 \AA$.

\begin{tabular}{|c|c|c|c|c|}
\hline Site & $\begin{array}{r}\mathrm{Fe}_{1} \\
\mathrm{Fe}-I^{\mathrm{bcc}}\end{array}$ & $\mathrm{Fe}-I I^{?}$ & $\mathrm{Fe}-I I I^{\mathrm{bcc}}$ & $\mathrm{N}$ \\
\hline Local symmetry & $D_{2 d}(4 d)$ & $C_{4 \mathrm{v}}(4 e)$ & $C_{1 h}(8 h)$ & $D_{4 h}(2 a)$ \\
\hline LSDA moment ${ }^{\mathrm{a}}\left(\mu_{B}\right)$ & 2.82 & 2.04 & 2.33 & 0.0 \\
\hline Fig. 3 moment at $U=1 \mathrm{eV}\left(\mu_{B}\right)$ & 2.8 & 2.0 & 2.4 & 0.0 \\
\hline Hyperfine field (Expt.) ${ }^{\mathrm{b}}(\mathrm{T})$ & 41.8 & 30.7 & 32.9 & \\
\hline Fermi contact field ${ }^{\mathrm{c}}(\mathrm{T})$ & 33.4 & 24.1 & 24.3 & \\
\hline Nearest $\mathrm{N}$ distance $(\AA)$ & $3.26(4)$ & $1.95(1)$ & $2.02(1)$ & $5.12(8)$ \\
\hline Nearest Fe shell at $(\AA)$ & 2.56 (III) & 2.35 (III) & 2.35 (II) & 1.95 (II) \\
\hline Next-nearest Fe shell at $(\AA)$ & 2.88 (II) & 2.39 (II) & $2.56(\mathrm{I})$ & 2.02 (III) \\
\hline Fe neighbors within $3.5 \AA$ & $(8,4,2)$ & $(4,1,4,4)$ & $(2,4,2,4,2)$ & $(2,4,8)$ \\
\hline
\end{tabular}

\begin{tabular}{lccc} 
& \multicolumn{2}{c}{$\mathrm{Fe}_{4} \mathrm{~N}$} & \\
Site & $\mathrm{Fe}^{\mathrm{fcc}}$ & $\mathrm{Fe}-I^{\mathrm{fcc}}$ & $\mathrm{N}$ \\
\hline Local symmetry $^{\mathrm{fcc}}$ & $O_{h}(1 a)$ & $D_{4 h}(3 c)$ & $O_{h}(1 a)$ \\
LSDA moment $^{\mathrm{a}}\left(\mu_{B}\right)$ & 2.98 & 2.23 & 0.0 \\
Fig. 3 moment at $U=1 \mathrm{eV}\left(\mu_{B}\right)$ & 3.08 & 2.39 & 0.0 \\
Experimental moment $^{\mathrm{d}}\left(\mu_{B}\right)$ & 2.98 & 2.01 & \\
Hyperfine field $^{\mathrm{b}}(\mathrm{T})$ & 36.9 & 23.5 & \\
Fermi contact field $^{\mathrm{c}}(\mathrm{T})$ & 36.3 & 21.7 & \\
Nearest N distance $\left.(\AA)^{(}\right)$ & $3.29(8)$ & $1.90(2)$ & $3.80(6)$ \\
Nearest Fe shell at $(\AA)$ & $2.68(\mathrm{II})$ & $2.68(\mathrm{I})$ & $1.90(\mathrm{II})$ \\
Next-nearest Fe shell at $(\AA)$ & $3.80(\mathrm{I})$ & $3.80(\mathrm{II})$ & $3.29(\mathrm{I})$ \\
Fe neighbors within 3.5 $\AA$ & $(12)$ & $(12)$ & $(6,8)$ \\
\hline
\end{tabular}

${ }^{\text {a } R e f e r e n c e ~} 16$ and this work.

${ }^{\mathrm{b}}$ Reference 9.

${ }^{\mathrm{c}}$ Reference 16.

${ }^{\mathrm{d}}$ Reference 12 .

$\mathrm{Fe}_{16} \mathrm{~N}_{2}$ phase. Central to converting these to site moments are issues associated with site symmetry. Note that the experimental values for $\mathrm{Fe}_{4} \mathrm{~N}$ in Table I come from neutrondiffaction studies ${ }^{12}$ and comparison with $\mathrm{Fe}_{4} \mathrm{~N}$ 's hyperfine fields lends credence to the value $3.5 \mu_{B}$ for $\mathrm{Fe}-\mathrm{I}$ in $\mathrm{Fe}_{16} \mathrm{~N}_{2}$.

\section{VOLUME EFFECTS AND LSDA RESULTS}

It is tempting to suggest that the experimental trends seen in the site moments are simply volume effects. The WignerSeitz (WS) volumes per atom do seem to correlate with experimental and predicted magnetic moments in certain rareearth-iron nitrides. ${ }^{13}$ However, for the situation at hand it provides only a zeroth-order picture of the experimental results. When Wigner-Seitz volumes are calculated for different sites of the two compounds at observed lattice parameters, the large moment site in $\mathrm{Fe}_{4} \mathrm{~N}$ turns out to have the largest WS volume, followed by the similar site in $\mathrm{Fe}_{16} \mathrm{~N}_{2}$. These volumes for $\mathrm{Fe}_{4} \mathrm{~N}$ are 12.7 and $13.65 \mathrm{~nm}^{3}$, and for $\mathrm{Fe}_{16} \mathrm{~N}_{2}$ they are $11.75,12.4$, and $12.9 \mathrm{~nm}^{3}$. If the moments scale with volume only, the large moment site in $\mathrm{Fe}_{4} \mathrm{~N}$ should carry the largest moment for both compounds, which is not the observed result. The band structure does play a role in reducing the volume (only) effects expected according to above scaling arguments. We have also carried out full potential LASTO (Ref. 19) calculations for these nitrides, as well as for a body-centered-tetragonal (bct) $\mathrm{Fe}_{8}$ structure with $\mathrm{Fe}$ atom positions the same as in $\mathrm{Fe}_{16} \mathrm{~N}_{2}$, but without nitrogen. Our results are in close agreement with other full potential calculations ${ }^{16}$ and these values are listed in Table I. In Fig. 2, our site projected densities of states (DOS) for nonmagnetic $\mathrm{Fe}_{16} \mathrm{~N}_{2}$ are shown. The DOS of two Fe sites (Fe-I and Fe-III) resemble bcc-type DOS, with the typical bcc minimum and maxima. This point of view is supported by the designations of WS polyhedra for various sites ${ }^{13}$ in $\mathrm{Fe}_{16} \mathrm{~N}_{2}$. We also note that the Fe-II and Fe-III DOS indicate a certain degree of interaction with nitrogen $p$ states, while for Fe-I, this interaction appears minimal, consistent with the fact that Fe-I and $\mathrm{N}$ are third nearest neighbors.

Most first-principles band-structure results based on LSDA predict that in $\mathrm{Fe}_{16} \mathrm{~N}_{2}$, the Fe-I site carries a moment comparable to, but smaller than in $\mathrm{Fe}_{4} \mathrm{~N}$ (Refs. 14-16) (see Table I). However, all of these calculated values have an upperbound of about $3.0 \mu_{B}$. A comparison of the nonmagnetic DOS of the two structures $\mathrm{Fe}_{8}$ and $\mathrm{Fe}_{16} \mathrm{~N}_{2}$ indicates that nitrogen induces a clear depletion of weight in the DOS near the Fermi level for the $\mathrm{Fe}$ site (Fe-II) closest to nitrogen. This is responsible for the comparatively low value of its 


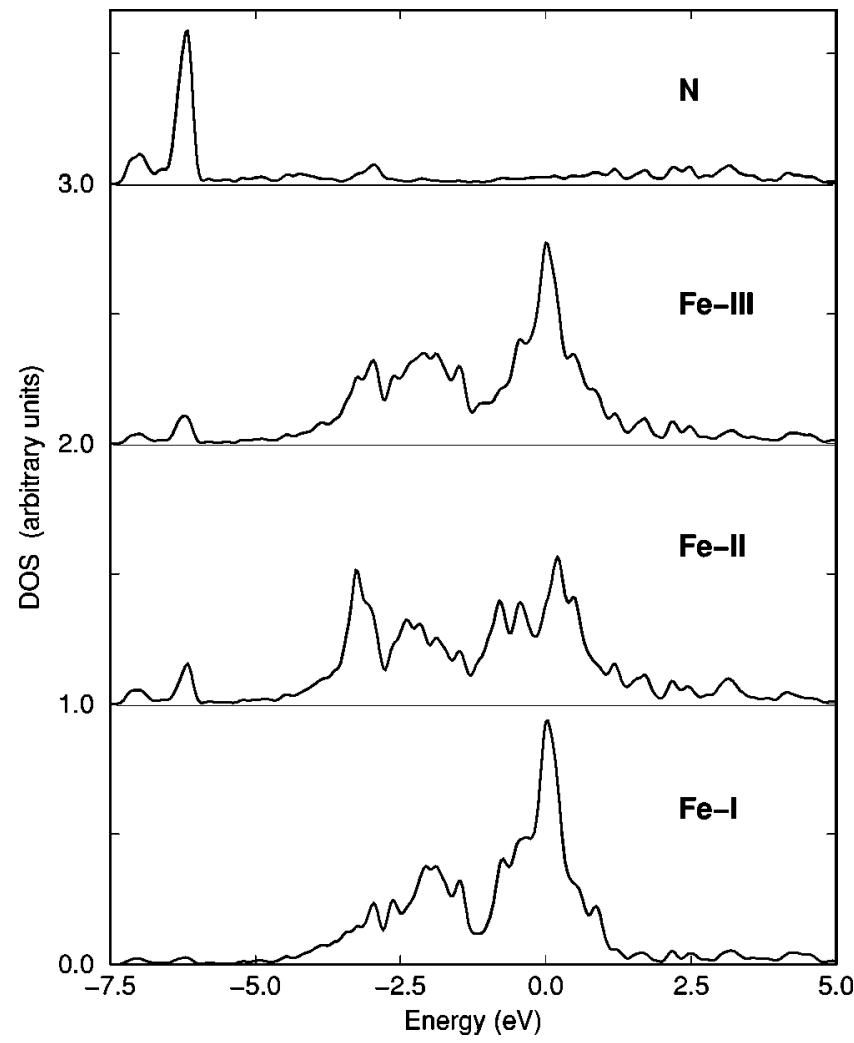

FIG. 2. Site projected densities of states (DOS) for nonmagnetic $\mathrm{Fe}_{16} \mathrm{~N}_{2}$. The top panel shows nitrogen DOS and the others (in descending order) show Fe-III, Fe-II, and Fe-I. These were calculated with $29 k$ points in the irreducible Brillouin zone.

magnetic moment seen in the calculations. For the remaining two sites (Fe-I and Fe-III), there are enhancements in DOS near the Fermi level. These results indicate that a structure equivalent to $\mathrm{Fe}_{16} \mathrm{~N}_{2}$ with the same unit cell volume but without nitrogen is unlikely to yield moments similar to those in $\mathrm{Fe}_{16} \mathrm{~N}_{2}$. As calculated in Ref. 16 and by us, the largest magnetic moment in the bct $\mathrm{Fe}_{8}$ crystal structure following nitrogen removal is only slightly larger than $2.5 \mu_{B}$, which is in fact smaller than for $\mathrm{Fe}_{16} \mathrm{~N}_{2}$ because of changes in DOS around the Fermi level. However, it is also clear that the magnetic moment for the corresponding site in $\mathrm{Fe}_{16} \mathrm{~N}_{2}$ as calculated within a full potential LSDA scheme does not exceed $3.0 \mu_{B}$.

\section{FERROMAGNETISM, THE HUBBARD MODEL, AND THE STONER APPROACH}

There are two main aspects of the interactions that have to be clarified in order to understand saturated ferromagnets. First, there has to be ferromagnetic coupling between sites in a given material and second, locally (i.e., at a given site), the intrasite interactions should favor saturation. Both these effects should coexist in order to have a strong ferromagnet.

Several many-particle effects may be responsible for the (possible) appearance of strong ferromagnetism in hard magnets based on $3 d$ metals, the first being a local Hund's-ruletype effect providing the maximum local magnetization due to exchange splitting in degenerate $3 d$ shells. The second is an enhanced electron polarization mechanism similar to the Stoner mechanism of saturated ferromagnetism in itinerant systems. This is often thought of as arising from local $d$ vs non- $d$ exchange contributions from spin polarization of other electrons polarized by the magnetic $3 d$ electrons. Another is the intra-atomic Coulomb repulsion $U$ between spin-up and spin-down electrons providing another Stoner-type exchange splitting and full spin alignment, as known in the Nagaoka problem. $^{20}$

Despite its simple appearance, the Hubbard model (in an external field $h$ )

$$
H=-t \sum_{\langle i j\rangle \sigma}\left(c_{i \sigma}^{+} c_{j \sigma}+(\text { H.c. })+U / 2 \sum_{i \sigma} n_{i \sigma} n_{i-\sigma}-h \sum_{i \sigma} \sigma n_{i \sigma}\right.
$$

has been extensively used to examine a wide variety of physical phenomena such as ferro- and antiferromagnetism, and the metal-insulator transition. Here $t$ denotes a hopping parameter, $U$ the on-site Coulomb repulsion, and $n_{i \sigma}$ the (occupation) number operator at site $i$ for spin $\sigma$. The above simple form describes relatively localized (tight binding) electrons with an explicit (on-site only) electron-electron interaction. There exist a considerable number of conflicting claims with regard to the magnetism associated with this model, and not many (related) exact results are known. In certain real materials one has to deal with an intermediate coupling $(U \sim t)$ regime and this is yet another complication for various approaches based upon the strong $(U \gg t)$ or weak $(U \ll t)$ coupling theories.

The behavior of magnetization of itinerant systems in an external field will help us understand how to build ferromagnetism in the ground state with $h=0$. The inclusion of an external field makes it possible to examine the fully polarized as well as the spin flipped states and thereby make predictions about the intermediate coupling regime. A systematic investigation of the above has been carried out by $\mathrm{Shiba}^{21}$ in the one-dimensional case, but the higher dimensional properties (at least for some materials) are expected to be quite different from those in one dimension. ${ }^{22}$

For an improved treatment of the Hubbard model, the interaction term involving $U$ can be decoupled using the most general decoupling scheme in the weak-coupling regime as

$$
\begin{aligned}
U n_{\uparrow} n_{\downarrow}= & U\left\langle n_{\uparrow}\right\rangle n_{\downarrow}+U n_{\uparrow}\left\langle n_{\downarrow}\right\rangle-U\left\langle n_{\uparrow}\right\rangle\left\langle n_{\downarrow}\right\rangle-U\left\langle c_{\uparrow}^{+} c_{\downarrow}\right\rangle c_{\downarrow}^{+} c_{\uparrow} \\
& -U\left\langle c_{\downarrow}^{+} c_{\uparrow}\right\rangle c_{\uparrow}^{+} c_{\downarrow}+U\left\langle c_{\uparrow}^{+} c_{\downarrow}\right\rangle\left\langle c_{\downarrow}^{+} c_{\uparrow}\right\rangle .
\end{aligned}
$$

Here on-site density-density correlations $\left(\left\langle n_{\uparrow}\right\rangle\left\langle n_{\downarrow}\right\rangle\right)$ as well as the electron-hole correlation parameter $\left(\Lambda=\left\langle c_{\uparrow}^{+} c_{\downarrow}\right\rangle\right)$ are explicitly included. These effects are related to each other and strongly depend on the values of $U$ and $t$, and hence can be drastically changed by different topologies (fcc, hcp, bcc, etc.), dimensionality, or variation of electron concentration. The most favorable conditions for the existence of ferromagnetism are expected for nonbipartite lattices away from halffilling where corresponding excitonic correlations are supressed $(\Lambda \rightarrow 0)$. By decreasing the magnetic field, the stabilization of the completely polarized state can occur for relatively strong $U$. At this level, the parameter $U$ represents an exchange interaction (as dictated by the the Hund's first rule in the atomic limit) and should be closely tied to a Stoner parameter. However, we note that these arguments 
can be extended to include orbital effects (for a given orbital angular momentum $l$ ) by incorporating a set of parameters $U_{\sigma, \sigma^{\prime}}^{m, m^{\prime}}$ within the above mean-field arguments, with $m, m^{\prime}$ denoting orbital magnetic quantum numbers.

Given a set of site projected densities of states for a nonmagnetic material, one can obtain straightforward estimates of site moments by employing simple Stoner-type arguments. As described above, the Stoner model employed here can be derived from a Hubbard model in the mean-field approximation. For a given value of the exchange splitting $\Delta$ one can calculate a (spin) moment by imposing the selfconsistency condition ${ }^{23}$

$$
\Delta=U \mu(\Delta),
$$

where $\mu$ is a function of the exchange splitting, and $U$ is related to the Stoner parameter $I .{ }^{24}$ This condition can be easily derived ${ }^{23}$ and should be regarded as a way to arrive at upperbounds to the possible on-site (local) moments, i.e., it is useful for looking into saturation moments, and provides physical insight.

Instead of using site projected DOS as described above, we have utilized the nonmagnetic wave-function information in the following way. Standard LDA calculations (with no spin polarization) were carried out using the full potential LASTO scheme ${ }^{19}$ for the three systems bcc Fe, $\mathrm{Fe}_{4} \mathrm{~N}$, and $\mathrm{Fe}_{16} \mathrm{~N}_{2}$ using their observed lattice parameters. A common muffin-tin radius of $2.2 a_{0}$ was used in all these calculations. Using these unpolarized eigenstates $\left(\psi_{0}^{i}\right)$ as the basis set at a given $k$ point, Hamiltonian matrix elements were evaluated within a Hubbard-Stoner scheme according to the following prescription:

$$
H_{i j}^{\sigma}=\epsilon_{0}^{i} \delta_{i j}+\left\langle\psi_{0}^{i}\left|\sum_{\alpha, l} \theta_{\alpha}(\mathbf{r}) \hat{\Delta}_{\alpha, l}^{\sigma}\right| \psi_{0}^{j}\right\rangle
$$

Here $\epsilon_{0}$ refers to the unpolarized LDA eigenvalue and the $\theta_{\alpha}$ function is defined to be equal to one inside the sphere $\alpha$ and zero elsewhere. Also $\hat{\Delta}_{\alpha, l}^{\sigma}=n_{\alpha, l}^{-\sigma} U_{\alpha, l} \hat{P}_{l}$ where $\hat{P}_{l}$ is a projection operator corresponding to orbital angular momentum $l$, and $n_{\alpha, l}^{-\sigma}$ denotes the integrated count of spin, opposite in sign to that of $\sigma$, contained in the sphere $\alpha$ for a given $l$. We note that if $n_{\alpha, l}^{\sigma}=n_{\alpha, l}^{-\sigma}$ for every atom and $l$, then the unpolarized system is recovered. This (polarized) Hamiltonian was diagonalized at all the $k$ points and site moments (differences in the above $n$ counts) were calculated selfconsistently using the same $U$ value for $d$ states in every Fe atom. Note that this procedure involves more than a rigid shift of unpolarized bands, since all the matrix elements (diagonal as well as off-diagonal) are updated at each step of the self-consistent procedure, i.e., changes in hybridization and charge transfer are allowed. The results were declared self-consistent when the input and output moments agreed to within $1 \times 10^{-4} \mu_{B}$.

\section{DISCUSSION}

The usual $U$ values found for metallic bcc Fe are about 1 $\mathrm{eV}$ (Ref. 24) (noting that $I$ as defined in Ref. 24, is $U / 2$ ). Figure 3 shows the values of the moments (found by selfconsistently solving the above Hamiltonian problem for a

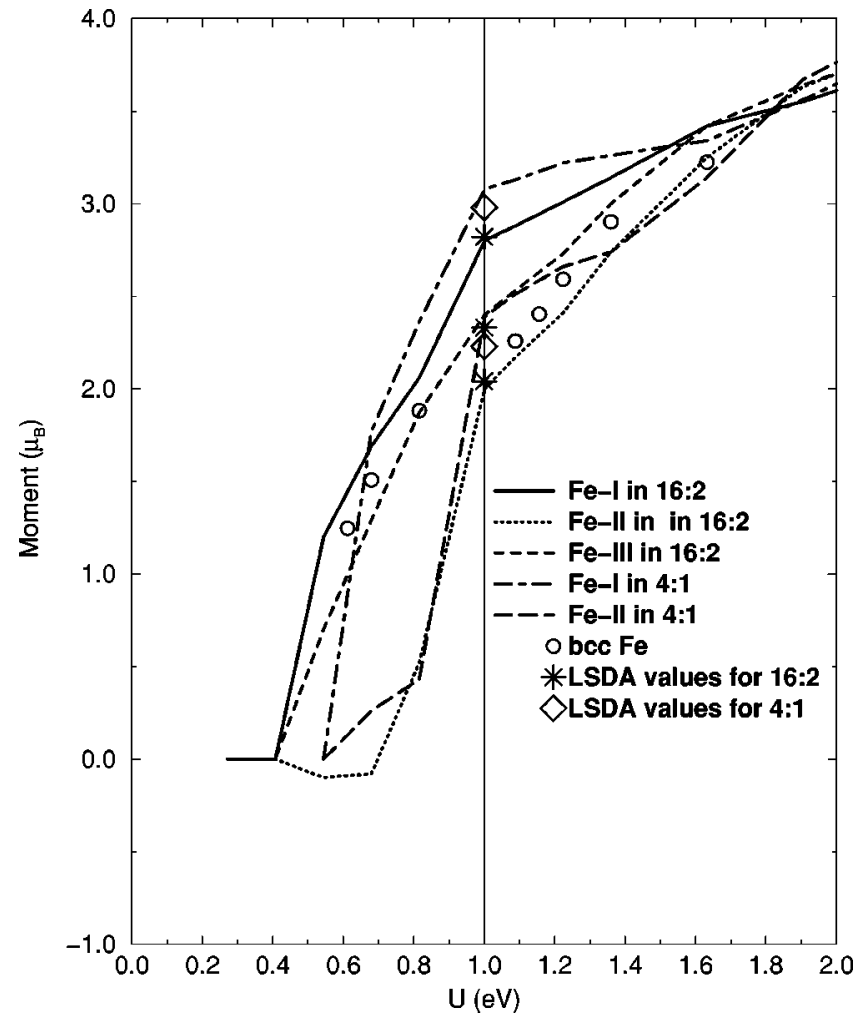

FIG. 3. Hubbard-Stoner derived moments vs $U$ as explained in the text for $\mathrm{Fe}_{16} \mathrm{~N}_{2}, \mathrm{Fe}_{4} \mathrm{~N}$, and bcc Fe. The appropriate $U$ value for (metallic) bcc Fe is about $1 \mathrm{eV}$. LSDA site moments for the two nitrides are also shown on this plot (at $U=1 \mathrm{eV}$ ) for comparison.

given $U$ ) as a function of $U$ for bcc $\mathrm{Fe}, \mathrm{Fe}_{4} \mathrm{~N}$, and $\mathrm{Fe}_{16} \mathrm{~N}_{2}$. For bcc Fe, we have calculated values of the moment for two different $k$-point sets, in order to examine the sensitivity to k-point sets (which turned out to be less than a tenth of a Bohr magneton). The most striking result emerging from Fig. 3 is that for $U$ values near $1 \mathrm{eV}$, the Stoner theory derived moments and band-structure results show good agreement. This is true for bcc Fe and also for the two nitrides under study. More importantly, this version of Hubbard-Stoner theory indicates that the $U$ values have to be unusually large, namely $\simeq 2 \mathrm{eV}$, in order to have site moments that are around $3.5 \mu_{B}$, for $\mathrm{Fe}_{4} \mathrm{~N}$ and $\mathrm{Fe}_{16} \mathrm{~N}_{2}$. Interestingly, even bcc Fe will produce similarly large magnetic moments at such high $U$ values. All the Fe sites in the two nitrides would carry comparable moments at such large $U$ values due to the high degree of saturation.

In both of these nitrides, the Fe-I site favors rapid polarization. Infact, the polarization behavior seems somewhat similar for each of the two sites, with the site in $\mathrm{Fe}_{4} \mathrm{~N}$ showing a slightly faster initial rate, while the site in $\mathrm{Fe}_{16} \mathrm{~N}_{2}$ eventually reaches a similar value for its moment. The bcc-like DOS in the latter is at least partly responsible for this behavior. The onset of the ferromagnetic instability correlates well with the Fe-N neighbors; i.e., the site that has nitrogen as the first (third) neighbor shows the weakest (strongest) onset. The sites nearest to $\mathrm{N}$ would require such large $U$ values for saturation due to the depletion of DOS near the Fermi level.

Experimental hyperfine fields and calculated Fermi contact field contributions are shown in Table I. The calculated contact fields using spin densities at the nuclei indicate a 
systematic error as discussed by Coehoorn, Daalderop, and Hansen, ${ }^{16}$ although they do follow the experimental trends. There are additional contributions to the hyperfine field not included in the calculations. As noted in the recent NMR experiment, reasonable values of proportionality constants $A$ (where \{hyperfine field at site $/ A=$ site moment) used to convert the hyperfine fields to magnetic moments range from $120 \mathrm{kOe} / \mu_{B}$ to $150 \mathrm{kOe} / \mu_{B}$. The values at the lower end are said to be appropriate for the nitrides with neutron scattering experiments ${ }^{12}$ on $\mathrm{Fe}_{4} \mathrm{~N}$ providing an independent source of support for this point of view. These values lead to an estimated moment of $3.5 \mu_{B}$ for the Fe-I site in $\mathrm{Fe}_{16} \mathrm{~N}_{2}$. However, there still remains the question of transferability of these proportionality constants to $\mathrm{Fe}_{16} \mathrm{~N}_{2}$. The values of $A$ at the upper end are normally used for bcc Fe. If these were used for $\mathrm{Fe}_{16} \mathrm{~N}_{2}$, the resulting magnetic moments would agree well with LSDA results. The moments so calculated would still be lower than those obtained from x-raydiffraction and magnetization measurements of Wallace and Huang $^{8}$ while agreeing with similar measurements of Takahashi and co-workers. ${ }^{10}$ However, as noted previously, these nitrides are a mixture of distorted bcc and fcc structures and from our calculated DOS shown in Fig. 2 and from the designations in Melamud, Bennett, and Watson, ${ }^{13}$ we may argue here that the environment of Fe-I and Fe-III sites in $\mathrm{Fe}_{16} \mathrm{~N}_{2}$ appear to resemble a bcc structure rather than a fcc one. From Table I, it is clear that the Fe atoms in $\mathrm{Fe}_{16} \mathrm{~N}_{2}$ are closer to one another than the fcc-like ones in $\mathrm{Fe}_{4} \mathrm{~N}$. The above factors as well as the effects due to $\mathrm{N}$ (lattice expansion and proximity to $\mathrm{Fe}$ ) play a crucial role in determining site moments.

\section{CONCLUSIONS}

A main conclusion from these Stoner arguments is that in order to obtain a saturation moment of $3.5 \mu_{B}$ for the iron sites under consideration, one has to resort to $U$ values that are measurably larger than the ones attributed to ordinary metallic iron; this may, in turn, imply somewhat unphysical charge transfers between sites. It would appear that, even with the Hubbard-Stoner approximation employed here, one is hard pressed to rationalize magnetic moments of the size which have been argued experimentally unless strong correlation effects are accommodated in these metallic systems.

On the theoretical front, if we were able to extract reliable
$U$ values from first principles for the 16:2 compound, it would have been straightforward to rule on the experiments that predict large Fe moments. However, this has turned out to be a nontrivial exercise. As mentioned previously, there are LDA+U-type calculations (Ref. 17) that predict large moments in this material using $U$ values calculated from an approach similar to constrained density functional (CDFT) calculations. In our opinion, their $U$ values (of about $4 \mathrm{eV}$ ) are quite large and unrealistic for the $\mathrm{Fe}-\mathrm{N}$ compounds that are metallic. The problem here (which will be the subject of a future report), is that the screening effects in metals are not properly taken into account in such CDFT calculations. ${ }^{25}$ For insulators, where screening effects are not that strong, the CDFT based $U$ values seem to work well. However, in transition metals, for example in bcc Fe, even the RPA screened $U$ values appear to be too high for calculating magnetic transition temperatures. ${ }^{26}$ Also note that the screening could be different for different channels or orbitals (such as $t_{2 g}$ and $e_{g}$ in a cubic environment). Although various attempts have been made for metals, in our opinion, there are no reliable first-principles methods for extracting $U$ values in itinerant or near itinerant systems. We conclude that there is a real need to search for a better way to obtain properly screened interactions in these materials from first principles.

On the experimental front, we understand that there are serious materials problems when growing $\mathrm{Fe}_{16} \mathrm{~N}_{2}$. In fact, some of the controversial aspects of this problem are probably closely tied to this issue. If relatively large and stable samples of $\mathrm{Fe}_{16} \mathrm{~N}_{2}$ single crystals can be grown, we suggest carrying out either neutron-scattering or magnetic-X-ray dichroism experiments as an independent way of measuring the magnetic moments in this compound.

\section{ACKNOWLEDGMENTS}

We thank Professor J. I. Budnick, Professor W. A. Hines, and Professor Y. D. Zhang for many useful discussions and T. Apostolova for assistance during the early stages of this work. This research was partially funded by the Research Foundation at the University of Connecticut and the Division of Materials Sciences, U.S. Department of Energy under Contract No. DE-AC02-98CH10886 and used resources of the National Energy Scientific Computing Center, which is supported by the DOE office of Energy Research.
${ }^{1}$ K. H. Jack, Proc. R. Soc. London, Ser. A 208, 200 (1951).

${ }^{2}$ T. K. Kim and M. Takahashi, Appl. Phys. Lett. 20, 492 (1972).

${ }^{3}$ K. Nakajima and S. Okamoto, Appl. Phys. Lett. 56, 92 (1990).

${ }^{4}$ M. Komuro, Y. Kozono, M. Hanazono, and Y. Sugita, J. Appl. Phys. 67, 5126 (1990).

${ }^{5}$ Y. Sugita, K. Mitsuoka, M. Momuro, H. Hoshiya, Y. Kozono, and M. Hanazono, J. Appl. Phys. 90, 5977 (1991).

${ }^{6}$ H. Takahashi, M. Mirusoka, M. Komuro, and Y. Sugita, J. Appl. Phys. 73, 6060 (1993).

${ }^{7}$ R. M. Metzger, X. Bao, and M. Carbucicchio, J. Appl. Phys. 76, 6626 (1994).

${ }^{8}$ W. E. Wallace and M. Q. Huang, J. Appl. Phys. 76, 6648 (1994).
${ }^{9}$ Y. D. Zhang, J. I. Budnick, W. A. Hines, M. Q. Huang, and W. E. Wallace, Phys. Rev. B 54, 51 (1996).

${ }^{10}$ H. Takahashi, H. Soji, and M. Takahashi, J. Magn. Magn. Mater. 174, 57 (1997); M. Takahashi, H. Takahashi, H. Nashi, H. Soji, T. Wakiyama, and M. Kuwabara, J. Appl. Phys. 79, 5564 (1996).

${ }^{11}$ J. M. D. Coey, Phys. World 6, 25 (1993); J. Appl. Phys. 76, 6632 (1994).

${ }^{12}$ B. C. Frazer, Phys. Rev. 112, 751 (1958).

${ }^{13}$ M. Melamud, L. H. Bennett, and R. E. Watson, J. Appl. Phys. 76, 6044 (1994).

14 A. Sakuma, J. Magn. Magn. Mater. 102, 127 (1991). 
${ }^{15}$ B. I. Min, Phys. Rev. B 46, 8232 (1992).

${ }^{16}$ R. Coehoorn, G. H. O. Daalderop, and H. J. F. Jansen, Phys. Rev. B 48, 3830 (1993).

${ }^{17}$ W. Y. Lai, Q. Q. Zheng, and W. Y. Hu, J. Phys.: Condens. Matter 6, L259 (1994).

${ }^{18}$ V. I. Anisimov, J. Zaanen, and O. K. Andersen, Phys. Rev. B 44, 943 (1991).

${ }^{19}$ G. W. Fernando, J. W. Davenport, R. E. Watson, and M. Weinert, Phys. Rev. B 40, 2757 (1989).

${ }^{20}$ Y. Nagaoka, Phys. Rev. 147, 392 (1966).

${ }^{21}$ H. Shiba, Phys. Rev. B 6, 930 (1972).
${ }^{22}$ G. W. Fernando, A. N. Kocharian, R. E. Watson, and M. Weinert, Physica B, 230-232, 509 (1997).

${ }^{23} \mathrm{M}$. Weinert and S. Blügel, in Magnetic Multilayers, edited by R. E. Watson and L. Bennett (World Scientific, Singapore, 1994); M. Weinert, S. Blügel, and P. D. Johnson, Phys. Rev. Lett. 71, 4097 (1993).

${ }^{24}$ J. F. Janak, Phys. Rev. B 16, 255 (1977).

${ }^{25}$ M. M. Steiner, R. C. Albers, and L. J. Sham, Phys. Rev. B 45, 13272 (1992).

${ }^{26}$ M. M. Steiner and J. W. Wilkins (unpublished). 